\title{
EFFECT OF CATECHOLAMINE-RECEPTOR STIMULATING AGENTS ON BLOOD PRESSURE AFTER LOCAL APPLICATION IN THE NUCLEUS TRACTUS SOLITARII OF THE MEDULLA OBLONGATA
}

\author{
PIETER ZANDBERG *, WYBREN DE JONG and DAVID DE WIED \\ Rudolf Magnus Institute for Pharmacology, Medical Faculty, University of Utrecht, \\ Vondellaan 6, 3521 GD Utrecht, The Netherlands
}

Received 20 November 1978, accepted 14 December 1978

P. ZANDBERG, W. DE JONG and D. DE WIED, Effect of catecholamine-receptor stimulating agents on blood pressure after local application in the nucleus tractus solitarii of the medulla oblongata, European J. Pharmacol. 55 (1979) 43-56.

The effect of various catecholamines and $\alpha$-mimetics, given by microinjection in the $\mathrm{A}_{2}$-region of the nucleus tractus solitarii (NTS), on blood pressure was investigated in anesthetized male rats. A dose-dependent decrease of blood pressure and heart rate was induced by adrenaline as the most effective drug, followed by noradrenaline, dopamine, $\alpha$-methylnoradrenaline and octopamine. Ablation of the rostral or caudal part of the NTS, or removal of the area postrema did not diminish the effect of $\alpha$-methylnoradrenaline. Higher doses of noradrenaline and $\alpha$-methylnoradrenaline caused an initial rise of blood pressure, while the blood pressure lowering effect of noradrenaline was diminished, and that of $\alpha$-methylnoradrenaline and dopamine delayed. Isoprenaline and the $(+)$-stereoisomers of noradrenaline and $\alpha$-methylnoradrenaline were ineffective. The hypotensive effect of dopamine was not prevented by systemic injection of the dopamine $\beta$-hydroxylase inhibitor FLA 63 . Prior application of haloperidol, yohimbine and phentolamine antagonized the hypotensive response to dopamine and $\alpha$-methylnoradrenaline. Application of peripherally effective $\alpha$-mimetics into the $A_{2}$-region had no or little effect, while high doses increased blood pressure. Tyramine and clonidine caused some decrease of blood pressure. Clonidine also decreased blood pressure when it was applied in the area of the locus coeruleus. Application of isoprenaline in the locus coeruleus also decreased blood pressure while in contrast adrenaline, noradrenaline, dopamine and $\alpha$-methylnoradrenaline increased blood pressure. The present data suggest that the catecholaminergic receptors in the $A_{2}$-region of the NTS differ from the classic vascular $\alpha$-receptor and that the NTS also may contain structures which can antagonize the decrease in blood pressure.

Catecholamines $\quad \alpha$-Mimetics $\quad$ Decrease of blood pressure $\quad$ Nucleus tractus solitarii

Locus coeruleus

\section{Introduction}

There is ample evidence that brain catecholamines play a role in cardiovascular control (see Chalmers, 1975; De Jong et al., 1975; Van Zwieten, 1975). The lower brain stem has been proposed as the primary site of

\footnotetext{
* Present address: National Institute of Public Health, Pharmacology, Laboratory P.O.Box 1, 3720 BA Bilthoven, The Netherlands.
}

action of the blood pressure lowering effect of L-dopa, $\alpha$-methyldopa and clonidine (for review see Henning, 1973, 1975; Van Zwieten, 1975; Laubie et al., 1977). It has been suggested that these drugs act by direct noradrenergic receptor stimulation and may mimic baroreceptor activation (Sinha et al., 1973; Schmitt et al., 1973; Haeusler, 1975; Kobinger and Pichler, 1975). Although no catecholaminergic synapses have yet been shown to exist in the baroreceptor pathway itself (Kobinger and Pichler, 1975; Haeusler, 
1977), catecholamines appear to modulate the tonic inhibitory control and reflex regulation of cardiovascular functions. The nucleus tractus solitarii (NTS) of the medulla oblongata is one of the brain-stem sites which appear to play a role in the effects of catecholamines on blood pressure. This nucleus, which receives the major part of the afferent sino-aortic baroreceptor fibres (see Palkovits and Záborszky, 1977 ), is densely innervated by catecholaminergic terminals and contains catecholaminergic cell bodies (Dahlström and Fuxe, 1964; Fuxe, 1965; Palkovits and Jacobowitz, 1974). Previous studies showed that local bilateral application of adrenaline, noradrenaline and $\alpha$-methylnoradrenaline in the NTS caused a decrease of blood pressure and heart rate (De Jong, 1974; Nijkamp and De Jong, 1975; De Jong and Nijkamp, 1976). The $A_{2}$ region (described by Dahlström and Fuxe 1964) was the part of the N'TS in which injections of $\alpha$-methylnoradrenaline were found to be most effective (Zandberg and De Jong, $1977 a)$. This region contains catecholaminergic cell bodies (Dahlström and Fuxe, 1964; Palkovits and Jacobowitz, 1974) and has high concentrations of dopamine, noradrenaline (Versteeg et al., 1976) and adrenaline (Van der Gugten et al., 1976).

In the present study, microinjections into the $A_{2}$-region were used to study the effect of dopamine, noradrenaline, adrenaline and other catecholaminergic substances on blood pressure and heart rate, in order to obtain more precise information about the receptor involved in this effect. Preliminary experiments with local microinjections of catecholaminergic agents in the locus coeruleus are reported as well.

\section{Materials and methods}

Male rats of an inbred Wistar strain (Wi/ cpb TNO, Zeist The Netherlands), weighing $200-250 \mathrm{~g}$ were anesthetized with urethane $(1.2 \mathrm{~g} / \mathrm{kg}$ i.p.). Blood pressure was recorded from the femoral artery with a polyethylene $\left(\mathrm{PE}_{50}\right)$ cannula and a Statham pressure trans- ducer (Model P23 Db) connected to a Grass polygraph (model 79). The blood pressure was calculated as diastolic pressure $+1 / 3$ (systolic pressure minus diastolic pressure). The heart rate was computed from the blood pressure pulse wave by means of a biotachometer (Narco-Bio systems, BT 1200).

The rats were placed in a stereotactic apparatus and the head flexed to an angle of $45^{\circ}$ downward for the injections into the NTS. After a midline incision through the doral neck muscles and opening of the atlantooccipital membrane the dorsal surface of the lower brain-stem was exposed via a limited occipital craniotomy using forceps. Microinjections in the area of the NTS were given through a glass cannula (outer diameter 60 $\mu \mathrm{m}$ ) in a volume of $0.4 \mu \mathrm{l}$, which was delivered in $10 \mathrm{sec}$ with an Agla micrometer syringe and a Sharlow micrometer. Except where otherwise indicated, the injections in the $A_{2}$-region were made unilaterally at the right side of the medulla at the level of the caudal tip of the area postrema, just medial of the tractus solitarius $(0.5 \mathrm{~mm}$ lateral of the midline and $0.9 \mathrm{~mm}$ below the dorsal surface of the medulla oblongata) as described previously (Zandberg and De Jong, 1977a). A localization study with $\alpha$-methylnoradrenaline showed that this was the most effective region of the NTS for producing a decrease in blood pressure. This plane, which corresponds to P $7.4 \mathrm{~mm}$ of the atlas of Palkovits an' bowitz (1974) and which is about $\iota \mathrm{m}$ caudal of the level of the obex, was the iore taken as the rostro-caudal zero. Each rat received only one injection, unless otherwise indicated.

In one experiment a microinjection of 0.3 nmoles ${ }^{14} \mathrm{C}$-noradrenaline $(39,000 \mathrm{dpm})$ in 0.4 $\mu \mathrm{l}$ saline was given. The distribution of the radioactivity was determined 5 min after the injection. The rats were decapitated, the brain removed from the skull and directly frozen on dry ice. Frozen sections $(100 \mu \mathrm{m})$ were cut in a cryostat. The radioactivity of the sections was determined in a liquid scintillation counter. More than $80 \%$ of the radioactivity 
recovered in the medulla oblongata was within the rostro-caudal distribution of 1200 $\mu \mathrm{m}$. A very similar distribution along the NTS has been observed with 2 nmoles ${ }^{14} \mathrm{C}$-clonidine (35 $800 \mathrm{dpm})$ in $0.4 \mu \mathrm{l}$ and with methylene blue (Zandberg and De Jong, 1977a,b).

Bilateral lesions with a diameter of about $800 \mu \mathrm{m}$ were made in the NTS with a stainless steel electrode (outer diameter $200 \mu \mathrm{m}$ ) with a bare tip of $200 \mu \mathrm{m}$. Current was applied through a radio frequency lesion generator (Radionics, model RFG-4) ( $13 \mathrm{~V}$ for $\mathrm{min}$ ). Surgical removal of the area postrema was performed under magnification in rats anesthetized with ether, $48 \mathrm{~h}$ before the experiment. A curved fine needle tip was used to excise the area postrema in a rostral direction. Care was taken to avoid damage to the cerebellum and the surrounding medullary structures.

For the injections in the locus coeruleus the heads of the rats were placed in the horizontal plane, as described by König and Klippel (1963). A hole was drilled on the right and left side in the skull and bilateral injections in a volume of $1.0 \mu$ l were made via a stainless steel injection needle (outer diameter $200 \mu \mathrm{m})$. The coordinates used were $2.5 \mathrm{~mm}$ posterior of lambda and $1.2 \mathrm{~mm}$ lateral of the midline and the depth was 7.0 $\mathrm{mm}$ below the surface of the skull.

For comparison the peripheral $\alpha$-mimetic activity of the $\alpha$-adrenergic drugs was determined in the pithed rat. The rats were anesthetized with ether and pithed through the orbit. Artificial respiration with a Palmer pump (68 strokes/min, tidal volume $3 \mathrm{ml}$ ) was applied and blood pressure was recorded (see above) from a carotid artery. Drugs were injected into the jugular vein in a volume of $0.1 \mathrm{ml}$ and immediately washed in with $0.1 \mathrm{ml}$ of $0.9 \% \mathrm{NaCl}$. Pressor responses were calculated as the increase in mean blood pressure at the peak of the pressor response. The body temperature of all rats was kept at $35-37^{\circ} \mathrm{C}$ with an infrared heating lamp. For the reference drug, (-)-noradrenaline, the dose causing an increase of $50 \mathrm{~mm} \mathrm{Hg}$ in mean blood pressure was 0.23 nmoles. Taking the potency of $(-)$-noradrenaline as 100 , the relative potencies were (-)-adrenaline $400 ;(-)-\alpha$-methylnoradrenaline 33 ; oxymetazoline 8 ; clonidine 3 ; ( \pm )-phenylephrine 3 ; dopamine $1 ;( \pm)$-octopamine 0.25 ; tyramine 0.5 and $( \pm)$-methoxamine 0.08 .

After termination of the experiments the rats were killed by decapitation. The brain was removed from the skull and fixed in $4 \%$ formalin. Frozen sections $(60 \mu \mathrm{m})$ were cut and stained with $0.1 \%$ thionine, after which the lesions and needle tracks were observed microscopically. The lesions and injections in the NTS and in the locus coeruleus were projected in an atlas, modified according to Palkovits and Jacobowitz (1974).

Data are reported as means \pm S.E.M. Student's $t$-test was used for statistical analysis of the results.

The following drugs, dissolved or diluted in saline, were used for local injections into the brain: (-)-adrenaline bitartrate (Sigma); (-)amidephrine mesylate; clonidine $\mathrm{HCl}$ (Boehringer Ingelheim), dopamine $\mathrm{HCl}$ (KochLight); haloperidol (Serenase ${ }^{\circledR}$, Janssen Pharmaceutica); (-)-isoprenaline sulphate (Sigma); $( \pm)$-methoxamine $\mathrm{HCl}$ (Burroughs Wellcome); (-)- $\alpha$-methylnoradrenaline $\mathrm{HCl}$ (SterlingWinthrop); (+)- $\alpha$-methylnoradrenaline (Sterling-Winthrop); (-)-noradrenaline bitartrate (Sigma); (+)-noradrenaline $\mathrm{HCl}$ (Sterling Winthrop); L-methyl $-{ }^{14} \mathrm{C}$-noradrenaline D-bitartrate (Radiochemical Centre, Amersham); ( \pm )-octopamine $\mathrm{HCl}$ (Sigma); oxymetazoline $\mathrm{HCl}$ (Merck); phentolamine (Regitine $\left.{ }^{\circledR}, \mathrm{Ciba}\right)$; ( \pm )-phenylephrine $\mathrm{HCl}$ (Koch-Light); tyramine $\mathrm{HCl}$ (Koch-Light); yohimbine $\mathrm{HCl}$ (Sigma). FLA 63, bis-(4-methyl-1-homopiperoizinyl thiocarbonyl)disulphate (Labkemi $\mathrm{AB}$ ) and urethane were injected intraperitoneally.

\section{Results}

\subsection{Comparison of unilateral and bilateral microinjections of $\alpha$-methylnoradrenaline}

Unilateral administration of $(-)$ - $\alpha$-methylnoradrenaline in the $A_{2}$-region of the NTS 
TABLE 1

Effect of unilateral injection of various doses of $(-)$-adrenaline, $(-)$-noradrenaline, dopamine and $(-)-\alpha-m e t h$ ylnoradrenaline in the $A_{2}$-region of the nucleus tractus solitarii (NTS) on mean blood pressure and heart rate. $n=$ number of rats.

\begin{tabular}{|c|c|c|c|c|c|c|}
\hline \multirow[t]{2}{*}{ Doses } & \multirow[t]{2}{*}{$\mathrm{n}$} & \multirow{2}{*}{$\begin{array}{l}\text { Mean } \\
\text { blood } \\
\text { pressure } \\
\text { (mm Hg) }\end{array}$} & \multicolumn{4}{|c|}{$\Delta$ Blood pressure after } \\
\hline & & & $2 \min$ & $5 \mathrm{~min}$ & $10 \mathrm{~min}$ & $15 \mathrm{~min}$ \\
\hline Saline & 13 & $116 \pm 3^{1}$ & $-2 \pm 1$ & $-2 \pm 1$ & $-2 \pm 1$ & $-2 \pm 1$ \\
\hline \multicolumn{7}{|c|}{ (-)-Adrenaline } \\
\hline 0.02 nmoles & 7 & $117 \pm 4$ & $-7 \pm 3$ & $-5 \pm 2$ & $-4 \pm 3$ & $-3 \pm 4$ \\
\hline 0.08 nmoles & 5 & $120 \pm 4$ & $-24 \pm 6^{4}$ & $-17 \pm 5^{4}$ & $-8 \pm 6$ & $-2 \pm 4$ \\
\hline 0.32 nmoles & 6 & $127 \pm 5$ & $-23 \pm 5^{4}$ & $-21 \pm 3^{4}$ & $-23 \pm 4^{4}$ & $-18 \pm 4^{4}$ \\
\hline 1.25 nmoles & 5 & $114 \pm 9$ & $-19 \pm 4^{4}$ & $-18 \pm 1^{4}$ & $-17 \pm 5^{4}$ & $-11 \pm 4^{3}$ \\
\hline \multicolumn{7}{|c|}{ (-)-Noradrenaline } \\
\hline 0.08 nmoles & 4 & $106 \pm 3$ & $-10 \pm 4^{3}$ & $-1 \pm 2$ & $+2 \pm 2$ & $+2 \pm 2$ \\
\hline $0.32 \mathrm{nmoles}$ & 6 & $132 \pm 4$ & $-27 \pm 4^{4}$ & $-13 \pm 3^{4}$ & $-9 \pm 3^{3}$ & $-2 \pm 2$ \\
\hline 1.25 nmoles & 8 & $120 \pm 2$ & $-20 \pm 1^{4}$ & $-15 \pm 2^{4}$ & $-11 \pm 3^{4}$ & $-9 \pm 2^{3}$ \\
\hline 5 nmoles & 5 & $113 \pm 5$ & $+2 \pm 11$ & $-7 \pm 8$ & $-12 \pm 6^{2}$ & $-15 \pm 4^{4}$ \\
\hline \multicolumn{7}{|l|}{ Dopamine } \\
\hline 0.32 nmoles & 6 & $125 \pm 4$ & $-15 \pm 5^{3}$ & $-11 \pm 3^{3}$ & $-6 \pm 4$ & $-4 \pm 5$ \\
\hline 1.25 nmoles & 7 & $121 \pm 2$ & $-15 \pm 2$ & $-20 \pm 3^{4}$ & $-14 \pm 4^{4}$ & $-11 \pm 2$ \\
\hline 5 nmoles & 5 & $109 \pm 5$ & $-19 \pm 3^{4}$ & $-22 \pm 4^{4}$ & $-21 \pm 5^{4}$ & $-13 \pm 6^{2}$ \\
\hline 20 nmoles & 7 & $121 \pm 6$ & $0 \pm 2$ & $-17 \pm 4^{4}$ & $-32 \pm 3^{4}$ & $-30 \pm 3^{4}$ \\
\hline \multicolumn{7}{|c|}{ (-)- $\alpha$-Methylnoradrenaline } \\
\hline 0.32 nmoles & 8 & $110 \pm 3$ & $-14 \pm 3^{4}$ & $-8 \pm 2^{3}$ & $0 \pm 2$ & $+3 \pm 2$ \\
\hline 1.25 nmoles & 7 & $110 \pm 2$ & $-18 \pm 2^{4}$ & $-20 \pm 4^{4}$ & $-16 \pm 4^{4}$ & $-10 \pm 3^{3}$ \\
\hline 5 nmoles & 9 & $111 \pm 3$ & $-19 \pm 3^{4}$ & $-27 \pm 3^{4}$ & $-25 \pm 3^{4}$ & $-21 \pm 3^{4}$ \\
\hline 20 nmoles & 13 & $129 \pm 2$ & $-3 \pm 2$ & $-25 \pm 2^{4}$ & $-31 \pm 1^{4}$ & $-32 \pm 2^{4}$ \\
\hline 80 nmoles & 5 & $114 \pm 5$ & $+7 \pm 3$ & $-8 \pm 6$ & $-34 \pm 4^{4}$ & $-34 \pm 4^{4}$ \\
\hline \multirow[t]{2}{*}{ Doses } & \multirow[t]{2}{*}{$\mathbf{n}$} & \multirow{2}{*}{$\begin{array}{l}\text { Heart rate } \\
\text { (bpm) }\end{array}$} & \multicolumn{4}{|c|}{$\Delta$ Heart rate after } \\
\hline & & & $2 \min$ & $5 \mathrm{~min}$ & $10 \mathrm{~min}$ & $15 \mathrm{~min}$ \\
\hline Saline & 13 & $444 \pm 12$ & $-14 \pm 10$ & $-9 \pm 8$ & $-1 \pm 4$ & $+2 \pm 4$ \\
\hline \multicolumn{7}{|c|}{ (-)-Adrenaline } \\
\hline 0.02 nmoles & 7 & $431 \pm 18$ & $-26 \pm 13$ & $-17 \pm 7$ & $-14 \pm 7$ & $-16 \pm 7$ \\
\hline 0.08 nmoles & 5 & $421 \pm 21$ & $-85 \pm 22^{4}$ & $-40 \pm 10^{2}$ & $-8 \pm 9$ & $-11 \pm 11$ \\
\hline 0.32 nmoles & 6 & $404 \pm 9$ & $-67 \pm 17^{3}$ & $-45 \pm 8^{3}$ & $-38 \pm 7^{4}$ & $-36 \pm 7^{4}$ \\
\hline 1.25 nmoles & 5 & $445 \pm 12$ & $-77 \pm 26^{3}$ & $-53 \pm 12^{3}$ & $-54 \pm 19^{3}$ & $-27 \pm 13^{3}$ \\
\hline \multicolumn{7}{|c|}{ (-)-Noradrenaline } \\
\hline 0.08 nmoles & 4 & $409 \pm 18$ & $-36 \pm 13$ & $-18 \pm 10$ & $-11 \pm 8$ & $-16 \pm 11$ \\
\hline 0.32 nmoles & 6 & $453 \pm 18$ & $-78 \pm 18^{3}$ & $-53 \pm 14^{3}$ & $-24 \pm 6^{3}$ & $-8 \pm 6$ \\
\hline 1.25 nmoles & 8 & $416 \pm 12$ & $-35 \pm 12$ & $-15 \pm 6$ & $-11 \pm 7$ & $-11 \pm 6$ \\
\hline 5 nmoles & 5 & $410 \pm 6$ & $-16 \pm 2$ & $-18 \pm 2$ & $-22 \pm 3^{3}$ & $-25 \pm 4^{4}$ \\
\hline \multicolumn{7}{|l|}{ Dopamine } \\
\hline 0.32 nmoles & 6 & $424 \pm 25$ & $-14 \pm 7$ & $-18 \pm 10$ & $-12 \pm 6$ & $-18 \pm 7^{2}$ \\
\hline 1.25 nmoles & 7 & $421 \pm 15$ & $-21 \pm 5$ & $-22 \pm 4$ & $-18 \pm 7^{2}$ & $-12 \pm 7^{3}$ \\
\hline 5 nmoles & 5 & $447 \pm 10$ & $-23 \pm 4$ & $-18 \pm 6$ & $-19 \pm 7^{2}$ & $-19 \pm 8^{2}$ \\
\hline 20 nmoles & 7 & $439 \pm 14$ & $-31 \pm 12$ & $-46 \pm 14$ & $-57 \pm 12^{4}$ & $-56 \pm 16^{4}$ \\
\hline
\end{tabular}


TABLE 1 (continued)

\begin{tabular}{|c|c|c|c|c|c|c|}
\hline \multirow[t]{2}{*}{ Doses } & \multirow[t]{2}{*}{$\mathrm{n}$} & \multirow{2}{*}{$\begin{array}{l}\text { Heart rate } \\
\text { (bpm) }\end{array}$} & \multicolumn{4}{|c|}{$\Delta$ Heart rate after } \\
\hline & & & $2 \mathrm{~min}$ & $5 \mathrm{~min}$ & $10 \mathrm{~min}$ & $15 \min$ \\
\hline \multicolumn{7}{|c|}{$(-)-\alpha-$ Methylnoradrenaline } \\
\hline 0.32 nmoles & 8 & $444 \pm 15$ & $-49 \pm 13^{2}$ & $-21 \pm 7$ & $-1 \pm 4$ & $+4 \pm 6$ \\
\hline 1.25 nmoles & 7 & $411 \pm 9$ & $-33 \pm 15$ & $-20 \pm 11$ & $-1 \pm 6$ & $+9 \pm 7$ \\
\hline 5 nmoles & 9 & $416 \pm 12$ & $-39 \pm 12$ & $-50 \pm 11^{3}$ & $-43 \pm 11^{4}$ & $-35 \pm 13^{3}$ \\
\hline 20 nmoles & 13 & $457 \pm 9$ & $-6 \pm 3$ & $-25 \pm 9$ & $-40 \pm 13^{2}$ & $-45 \pm 15^{3}$ \\
\hline 80 nmoles & 5 & $422 \pm 11$ & $-31 \pm 16$ & $-35 \pm 14$ & $-72 \pm 18^{4}$ & $-91 \pm 17^{4}$ \\
\hline
\end{tabular}

1 Mean \pm S.E.M.

$2 \mathrm{P}<0.05$.

${ }^{3} \mathrm{P}<0.01$.

${ }^{4} \mathrm{P}<0.001$.

caused a decrease of blood pressure (table 1). Maximal effects were reached within $10 \mathrm{~min}$ and were dose-dependent. The lowest dose which decreased blood pressure was 0.32 nmoles. With the 20 nmoles dose a slight increase in blood pressure $(6 \pm 1 \mathrm{~mm} \mathrm{Hg})$ was

TABLE 2

Effect of bilateral injections of various doses of (-)- $\alpha$-methylnoradrenaline in the $A_{2}$-region of the nucleus tractus solitarii (NTS) on mean blood pressure and heart rate. $n=$ number of rats.

\begin{tabular}{|c|c|c|c|c|c|c|}
\hline \multirow[t]{2}{*}{ Doses } & \multirow[t]{2}{*}{$\mathrm{n}$} & \multirow{2}{*}{$\begin{array}{l}\text { Mean } \\
\text { blood } \\
\text { pressure } \\
\text { (mm Hg) }\end{array}$} & \multicolumn{4}{|c|}{$\Delta$ Blood pressure after } \\
\hline & & & $2 \mathrm{~min}$ & $5 \mathrm{~min}$ & $10 \mathrm{~min}$ & $15 \mathrm{~min}$ \\
\hline Saline & 8 & $108 \pm 4^{1}$ & $-3 \pm 2$ & $0 \pm 2$ & $-1 \pm 3$ & $-1 \pm 3$ \\
\hline \multicolumn{7}{|c|}{ (-)- $\alpha$-Methylnoradrenaline } \\
\hline 0.02 nmoles & 5 & $115 \pm 4$ & $-5 \pm 2$ & $+1 \pm 1$ & $0 \pm 2$ & $-11 \pm 1$ \\
\hline 0.08 nmoles & 5 & $116 \pm 5$ & $-29 \pm 5^{4}$ & $-15 \pm 3^{3}$ & $-7 \pm 2$ & $-2 \pm 3$ \\
\hline 0.32 nmoles & 7 & $113 \pm 3$ & $-31 \pm 6^{4}$ & $-19 \pm 4^{3}$ & $-7 \pm 4$ & $-4 \pm 2$ \\
\hline 1.25 nmoles & 6 & $115 \pm 4$ & $-36 \pm 3^{4}$ & $-26 \pm 2^{4}$ & $-16 \pm 4^{3}$ & $-8 \pm 5$ \\
\hline \multirow[t]{2}{*}{ Doses } & \multirow[t]{2}{*}{$\mathrm{n}$} & \multirow{2}{*}{$\begin{array}{l}\text { Heart rate } \\
\text { (bpm) }\end{array}$} & \multicolumn{4}{|c|}{$\Delta$ Heart rate after } \\
\hline & & & $2 \mathrm{~min}$ & $5 \mathrm{~min}$ & $10 \mathrm{~min}$ & $15 \mathrm{~min}$ \\
\hline Saline & 8 & $446 \pm 13$ & $-46 \pm 17$ & $-28 \pm 10$ & $-18 \pm 8$ & $-16 \pm 9$ \\
\hline \multicolumn{7}{|c|}{ (-)- $\alpha$-Methylnoradrenaline } \\
\hline 0.02 nmoles & 5 & $436 \pm 26$ & $-23 \pm 24$ & $-2 \pm 13$ & $+2 \pm 10$ & $-6 \pm 16$ \\
\hline 0.08 nmoles & 5 & $421 \pm 20$ & $-80 \pm 12$ & $-42 \pm 17$ & $-8 \pm 11$ & $-6 \pm 12$ \\
\hline 0.32 nmoles & 7 & $458 \pm 13$ & $-57 \pm 18$ & $-22 \pm 15$ & $-1 \pm 10$ & $+5 \pm 8$ \\
\hline 1.25 nmoles & 6 & $408 \pm 19$ & $-96 \pm 18$ & $-81 \pm 8^{4}$ & $-59 \pm 10^{3}$ & $-53 \pm$ \\
\hline
\end{tabular}

1 Mean \pm S.E.M.

$2 \mathrm{P}<0.05$.

$3 \mathrm{P}<0.01$.

$4 \mathrm{P}<0.001$. 
observed directly after the injection (maximal after about $1 \mathrm{~min}$ ) and became more pronounced after 80 nmoles $(11 \pm 2 \mathrm{~mm} \mathrm{Hg})$. Bilateral injections of $(-)-\alpha$-methylnoradrenaline were more effective than unilateral ones in decreasing blood pressure (table 2). There were no pressor responses to $\alpha$-methylnoradrenaline after bilateral application of $0.02-1.25$ nmoles doses. The minial effective dose which significantly decreased blood pressure after bilateral application was 0.08 nmoles. A decrease of heart rate was observed after 5.0 nmoles given unilaterally and after 1.25 nmoles given bilaterally. Although the hypotensive response was more pronounced after bilateral injections (especially in the first 5-10 min) and bradycardia was observed with lower doses than after unilateral administration, there was no essential difference in the pattern of the response. Unilateral injections were used in subsequent experiments because the unilateral injection sites could be localized histologically more precisely. No difference was found in responses to injections (20 nmoles) given into the NTS in the left $(-25 \pm 5 \mathrm{~mm} \mathrm{Hg} ; \mathrm{n}=5)$ or right side $(-28 \pm 4 \mathrm{~mm} \mathrm{Hg} ; \mathrm{n}=5)$ of the medulla.

\subsection{Effective part of the NTS}

Lesions of the NTS just rostral of the obex level, which caused an increase in blood pressure did not diminish the blood pressure response to a unilateral application of $\alpha$-methylnoradrenaline in the $\mathrm{A}_{2}$ - region compared to the control group (table 3). After lesions in the nucleus commissuralis, which did not change blood pressure, $\alpha$-methylnoradrenaline caused a decrease in blood pressure as well. Removal of the area postrema also did not affect basal blood pressure or the blood pressure lowering effect. In rats bearing lesions or with the area postrema excised there was no change in basal and drug-induced alteration of heart rate.

\subsection{Effects of adrenaline, noradrenaline and dopamine}

Injection of (-)-adrenaline, ( -)-noradrenaline and dopamine into the $\mathrm{A}_{2}$-region also decreased blood pressure (table 1). A dose of (-)-adrenaline as low as 0.08 nmoles lowered blood pressure and heart rate. These decreases became more prolonged after 0.32 and 1.25 nmoles. An 0.08 nmole dose of (-)-nor-

\section{TABLE 3}

Effect of unilateral injection of 20 nmoles (-)- $\alpha$-methylnoradrenaline in the $A_{2}$-region of the nucleus tractus solitarii (NTS) on blood pressure and heart rate after bilateral lesions $1.0 \mathrm{~mm}$ rostral or caudal of zero level in the NTS or after surgical removal of the area postrema. $n=$ number of rats.

\begin{tabular}{lllll}
\hline & $\mathrm{n}$ & $\begin{array}{l}\text { Mean blood } \\
\text { pressure } \\
(\mathrm{mm} \mathrm{Hg})\end{array}$ & $\begin{array}{l}\Delta \text { blood } \\
\text { pressure } \\
(\mathrm{mm} \mathrm{Hg})\end{array}$ & $\begin{array}{l}\Delta \text { blood } \\
\text { pressure } \\
(\%)\end{array}$ \\
\hline Control & 5 & $120 \pm 31$ & $-24 \pm 2$ & 20.0 \\
Lesion 1.0 mm rostral of zero level $^{2}$ & 7 & $139 \pm 7$ & $-35 \pm 7$ & 25.2 \\
Lesion 1.0 mm caudal of zero level $^{2}$ & 5 & $112 \pm 4$ & $-18 \pm 5$ & 16.1 \\
Sham removal of area postrema $^{3}$ & 4 & $105 \pm 8$ & $-20 \pm 4$ & 19.0 \\
Removal of area postrema $^{3}$ & 6 & $103 \pm 3$ & $-20 \pm 2$ & 19.4 \\
\hline
\end{tabular}

1 Mean \pm S.E.M. Changes in blood pressure were measured $10 \mathrm{~min}$ after the injection.

2 The lesions were made 30 min before the injections.

3 The injections after removal of the area postrema were given $700 \mu \mathrm{m}$ caudal of zero level; in these rats the removal of the area postrema or the sham operation were performed $48 \mathrm{~h}$ prior to the experiment. 


\section{TABLE 4}

Effect of unilateral injection of various doses of $(-)$-isoprenaline, $(+)$-noradrenaline, $(+)-\alpha$-methylnoradrenaline, tyramine, $( \pm)$-octopamine, clonidine, $(-)$-amidephrine, $( \pm)$-methoxamine, $( \pm)$-phenylephrine and oxymetazoline in the $A_{2}$-region of the nucleus tractus solitarii (NTS) on mean blood pressure and heart rate. $n=$ number of rats.

\begin{tabular}{|c|c|c|c|}
\hline Doses & $\mathrm{n}$ & $\begin{array}{l}\Delta \text { Blood pressure } \\
(\mathrm{mm} \mathrm{Hg})\end{array}$ & $\begin{array}{l}\Delta \text { Heart rate } \\
(\mathrm{bpm})\end{array}$ \\
\hline
\end{tabular}

\section{Saline}

7

(-)-Isoprenaline

1.25 nmoles

5 nmoles

20 nmoles

80 nmoles

(+)-Noradrenaline

20 nmoles

80 nmoles

(+)- $\alpha$-Methylnoradrenaline

20 nmoles

200 nmoles

Tyramine

5 nmoles

20 nmoles

80 nmoles

(士)-Octopamine

1.25 nmoles

5 nmoles

20 nmoles

Clonidine

1.25 nmoles

5 nmoles

20 nmoles

80 nmoles

(-)-Amidephrine

5 nmoles

20 nmoles

80 nmoles

(士)-Methoxamine

5 nmoles

20 nmoles

80 nmoles

(士)-Phenylephrine

5 nmoles

20 nmoles

80 nmoles

Oxymetazoline

5 nmoles

20 nmoles
$-2 \pm 1^{1}$

$-7 \pm 6$

$$
\begin{array}{r}
-6 \pm 2 \\
-9 \pm 7 \\
-11 \pm 4 \\
+7 \pm 4
\end{array}
$$

$$
\begin{aligned}
& +12 \pm 2^{3} \\
& -8 \pm 3
\end{aligned}
$$$$
-8 \pm 3
$$$$
-21 \pm 4
$$$$
-54 \pm 10^{3}
$$

4
9

4
8

$+1 \pm 2$

$+7 \pm 3^{2}$

$-1 \pm 2$
$-3 \pm 5$

$-25 \pm 5$

$-43 \pm 21$

$\begin{array}{ll}5 & -16 \pm 3^{3} \\ 6 & -11 \pm 4^{2}\end{array}$

$\begin{array}{ll}5 & -16 \pm 3^{3} \\ 6 & -11 \pm 4^{2}\end{array}$

$-36 \pm 4^{3}$

$-4 \pm 5$

$-11 \pm 42$

$-25 \pm 9$

$-48 \pm 21$

$-21 \pm 3^{4}$

$-60 \pm 21^{2}$

$$
-21 \pm 3^{4}
$$

$\begin{aligned} 7 & -4 \pm 4 \\ 11 & -20 \pm 6^{2} \\ 11 & -11 \pm 6 \\ 3 & +30 \pm 5^{4}\end{aligned}$

$-31 \pm 20$

$-76 \pm 19^{2}$

$-47 \pm 10^{2}$

$-45 \pm 35$

\footnotetext{
1 Mean $\pm S$.
sure and heart
tables $1-3$.
$2 \mathrm{P}<0.05$.
${ }^{3} \mathrm{P}<0.01$.

$4 \mathrm{P}<0.001$.
} 
adrenaline decreased blood pressure while a 0.32 nmole dose also decreased heart rate. With a 5.0 nmole dose there was an initial rise of blood pressure $(12 \pm 5 \mathrm{~mm} \mathrm{Hg})$ directly after the injection (maximal after about $1 \mathrm{~min}$ ) and the subsequent decrease of blood pressure was less marked. Dopamine was about as effective as $\alpha$-methylnoradrenaline in decreasing blood pressure. There was a decrease in blood pressure with the 0.32 nmole dose. With the higher doses of dopamine the decrease became more prolonged and more pronounced and the heart rate also decreased. The 20 nmole dose decreased blood pressure after a delay of $2-5 \mathrm{~min}$.

The pattern of the decrease in blood pressure after dopamine and $\alpha$-methylnoradrenaline was different from that following adrenaline and noradrenaline. Adrenaline and noradrenaline caused a sharp drop in blood pres- sure, reaching a maximum within $5 \mathrm{~min}$, while the effect of $\alpha$-methylnoradrenaline and dopamine developed more slowly.

\subsection{Effects of various adrenergic substances}

As shown in table 4, various doses of ( - )isoprenaline were ineffective. The $(+)$-stereoisomers of noradrenaline and $\alpha$-methylnoradrenaline either did not alter the blood pressure or increased it.

Tyramine, 20 nmoles, induced a significant decrease in blood pressure and heart rate and 80 nmoles did not induce a significantly greater change in blood pressure. $( \pm)$-Octopamine, 5 and 20 nmoles, lowered blood pressure. The response pattern was similar to that with dopamine and $\alpha$-methylnoradrenaline. Oxymetazoline and $( \pm)$-phenylephrine were ineffective in changing blood pressure. Only the 20 nmole dose of $( \pm)$-methoxamine and

\section{TABLE 5}

Effect on mean blood pressure and heart rate of unilateral injection of 5 nmoles dopamine and 5 nmoles $(-)-\alpha$ methylnoradrenaline in the $\mathbf{A}_{2}$-region of the nucleus tractus solitarii (NTS) after pretreatment with FLA 63, or following administration of phentolamine, yohimbine and haloperidol at the same injection site. $n=$ number of rats.

\begin{tabular}{lllr}
\hline & $n$ & $\begin{array}{l}\Delta \text { Blood pressure } \\
(\mathrm{mm} \mathrm{Hg})\end{array}$ & $\begin{array}{l}\Delta \text { Heart rate } \\
(\mathrm{bpm})\end{array}$ \\
\hline $\begin{array}{l}\text { Dopamine } \\
\text { Saline }\end{array}$ & 3 & $-21 \pm 1^{1}$ & $-16 \pm 7$ \\
FLA 63 (25 mg/kg i.p.) & 5 & $-31 \pm 3$ & $-35 \pm 17$ \\
Phentolamine (5 nmoles) & 6 & $-1 \pm 2^{4}$ & $-30 \pm 6$ \\
Yohimbine (1.25 nmoles) & 4 & $-4 \pm 4^{3}$ & $-23 \pm 6$ \\
Haloperidol (5 nmoles) & 6 & $-3 \pm 5^{2}$ & $-9 \pm 5$ \\
Haloperidol (0.32 nmoles) & 5 & $-11 \pm 4$ & $-8 \pm 6$ \\
& & & $-11 \pm 12$ \\
(-)- $\alpha$-Methylnoradrenaline & & $-28 \pm 2$ & $-8 \pm 8$ \\
Saline & 4 & $-27 \pm 2$ & $-5 \pm 8$ \\
FLA 63 (25 mg/kg i.p.) & 5 & $-3 \pm 5^{4}$ & $+4 \pm 5$ \\
Phentolamine (5 nmoles) & 4 & $+6 \pm 6^{4}$ & $+2 \pm 6$ \\
Yohimbine (1.25 nmoles) & 5 & $+3 \pm 2^{4}$ & $-4 \pm 2$ \\
Haloperidol (5 nmoles) & 4 & $-14 \pm 6$ & \\
Haloperidol (0.32 nmoles) & 5 & & \\
\hline
\end{tabular}

\footnotetext{
${ }^{1}$ Mean \pm S.E.M. Changes in blood pressure were measured $10 \mathrm{~min}$ after the injection. Basal values for blood pressure and heart rate of the groups were not significantly different and were in the same range as those shown in Tables 1-3.

$2 \mathrm{P}<0.05$.

${ }^{3} \mathrm{P}<0.01$.

${ }^{4} \mathrm{P}<0.001$.
} 
( -)-amidephrine somewhat lowered the blood pressure while 5 nmoles clonidine could lower it. However, a higher dose, 80 nmoles, of clonidine and methoxamine only increased blood pressure. The maximal rise of blood pressure occurred within $5 \mathrm{~min}$. The heart rate was somewhat decreased by all these substances, especially with the higher doses (table $4)$.
3.5. Effects of $\alpha$-adrenoceptor blocking agents
and of systemic pretreatment with FLA 63

The $\alpha$-adrenergic blocking agents phentolamine ( 5 nmoles) and yohimbine $(1.25$ nmoles), locally applied to the $\mathrm{A}_{2}$-region $10 \mathrm{~min}$ previously blocked the effect of both 5 nmoles $\alpha$-methylnoradrenaline and 5 nmoles dopamine. Haloperidol (5 nmoles) also

\section{TABLE 6}

Effect on blood pressure and heart rate of bilateral injections of various doses of $(-)$-isoprenaline, $(-)$-adrenaline, $(-)$ - $\alpha$-methylnoradrenaline, $(-)$-noradrenaline, dopamine and clonidine in the locus coeruleus. $n=$ number of rats.

\begin{tabular}{|c|c|c|c|}
\hline Doses & $\mathrm{n}$ & $\begin{array}{l}\Delta \text { Blood pressure } \\
(\mathrm{mm} \mathrm{Hg})\end{array}$ & $\begin{array}{l}\Delta \text { Heart rate } \\
(\text { bpm })\end{array}$ \\
\hline Saline & 4 & $-1 \pm 3$ & $-7 \pm 3^{1}$ \\
\hline \multicolumn{4}{|c|}{ (-)-Isoprenaline } \\
\hline 1.25 nmoles & 5 & $-3 \pm 1$ & $-20 \pm 5$ \\
\hline 5 nmoles & 5 & $-13 \pm 10$ & $-19 \pm 6$ \\
\hline 20 nmoles & 6 & $-17 \pm 6^{2}$ & $-40 \pm 5^{4}$ \\
\hline \multicolumn{4}{|c|}{$(-)$-Adrenaline } \\
\hline 1.25 nmoles & 6 & $+9 \pm 3$ & $-4 \pm 3$ \\
\hline 5 nmoles & 6 & $+13 \pm 5$ & $-16 \pm 7$ \\
\hline 20 nmoles & 5 & $+5 \pm 8$ & $-37 \pm 8^{3}$ \\
\hline \multicolumn{4}{|c|}{$(-)-\alpha$-Methylnoradrenaline } \\
\hline 5 nmoles & 3 & $+14 \pm 2^{2}$ & $-7 \pm 7$ \\
\hline 20 nmoles & 5 & $+14 \pm 7$ & $-14 \pm 6$ \\
\hline \multicolumn{4}{|c|}{ (一)-Noradrenaline } \\
\hline 5 nmoles & 6 & $+22 \pm 4^{3}$ & $-17 \pm 6$ \\
\hline 20 nmoles & 6 & $+24 \pm 5^{3}$ & $-29 \pm 4^{3}$ \\
\hline \multicolumn{4}{|l|}{ Dopamine } \\
\hline 5 nmoles & 3 & $+8 \pm 5$ & $-7 \pm 7$ \\
\hline 20 nmoles & 6 & $+14 \pm 5^{2}$ & $-28 \pm 11$ \\
\hline \multicolumn{4}{|l|}{ Clonidine } \\
\hline 1.25 nmoles & 2 & $-18 \pm 3^{2}$ & $-25 \pm 5^{2}$ \\
\hline 5 nmoles & 5 & $-13 \pm 2^{3}$ & $-30 \pm 11$ \\
\hline 20 nmoles & 5 & $-30 \pm 10^{2}$ & $-66 \pm 21^{2}$ \\
\hline
\end{tabular}

\footnotetext{
${ }^{1}$ Mean \pm S.E.M. Changes in blood pressure were measured $10 \mathrm{~min}$ after the injection. Basal values for blood pressure and heart rate of the groups were not significantly different and were in the same range as those shown in tables $1-3$.

$2 \mathrm{P}<0.05$.

$3 \mathrm{P}<0.01$.

${ }^{4} \mathrm{P}<0.001$.
} 
blocked the blood pressure lowering response to 5 nmoles dopamine as well as to 5 nmoles $\alpha$-methylnoradrenaline and 0.32 nmoles still reduced the effect of both drugs to a similar extent (table 5). The blocking agents themselves did not significantly change blood pressure and heart rate.

The blood pressure lowering effect of $\alpha$-methylnoradrenaline and dopamine was not blocked by administration of FLA 63, given i.p. $1 \mathrm{~h}$ before the local injection (table 5 ). However there was some delay $(2-5 \mathrm{~min})$ before the decrease of blood pressure occurred.

\subsection{Effects of catecholamines and clonidine} after injection into the area of the locus coeruleus

As shown in table 6 (-)-adrenaline, (-)noradrenaline, dopamine and (-)- $\alpha$-methylnoradrenaline caused an increase in blood pressure after bilateral local application into the locus coeruleus. An injection of 5 nmoles noradrenaline into the fourth ventricle did not change the blood pressure or heart rate. (-)-Isoprenaline (20 nmoles) and clonidine, however, decreased blood pressure. Clonidine and the higher doses of adrenaline and noradrenaline also caused bradycardia.

\section{Discussion}

Our previous localization study with local injections of $\alpha$-methylnoradrenaline into the NTS of rats showed that the most effective region for causing a decrease in blood pressure was at the level of the caudal tip of the area postrema (Zandberg and De Jong, 1977a). Injections into the NTS just rostral of the obex level were ineffective, while injections caudally in the commissural part of the NTS were only slightly effective. These findings are in accordance with the present results, showing that bilateral lesions in the NTS just rostral of the obex, or in the nucleus commissuralis, or removal of the area postrema did not change the blood pressure lowering effect of $\alpha$-methylnoradrenaline when the same injection technique was used. Taken together, these results support the hypothesis that the $\mathrm{A}_{2}$-region is the site in the NTS where catecholamines cause a decrease in blood pressure and heart rate. The present microinjection studies did not allow a more precise localization since there was an elongated rostro-caudal distribution of the injection volume $(0.4 \mu \mathrm{l})$ along the NTS over a distance of approximately $1200 \mu \mathrm{m}$, as shown with radioactive noradrenaline and, previously, with methylene blue and ${ }^{14} \mathrm{C}$-clonidine (Zandberg and De Jong, 1977a,b). Interestingly these results indicate a differentiation between the area from which hypertension can be evoked by lesions and the area which, when injected with catecholamines, evokes a decrease of blood pressure, because only lesions of the NTS located rostral of the $A_{2}$ region elicited hypertension (Zandberg et al., 1978).

The relative potencies of the substances which, when applied to the $\mathrm{A}_{2}$-region, decreased blood pressure, were adrenaline $>$ noradrenaline $>$ dopamine $>\alpha$-methylnoradrenaline $>$ octopamine. Although bilateral injections were more effective than unilateral ones, the unilateral microinjection technique as used in the present study with a smaller cannula size and injection volume was apparently more sensitive than the bilateral injection technique used earlier (De Jong and $\mathrm{Nij}$ kamp, 1976). The more prolonged effect of $\alpha$-methylnoradrenaline and the fact that the maximal decrease of blood pressure induced by adrenaline and noradrenaline, occurred within 5 min may explain the fact that De Jong and Nijkamp (1976) had previously found $\alpha$-methylnoradrenaline somewhat more effective than adrenaline $5 \mathrm{~min}$ after administration.

The finding, that isoprenaline and the (+)stereoisomers of noradrenaline and $\alpha$-methylnoradrenaline were ineffective is consistent with the idea that the blood pressure lowering effect of the various catecholamines may be 
caused by direct stimulation of postsynaptic stereospecific $\alpha$-adrenoceptors in the brain (see Introduction). However, dopamine was effective in a dose-range similar to that of $\alpha$-methylnoradrenaline. Apart from an action on dopamine receptors, dopamine may stimulate $\alpha$ - and $\beta$-receptors directly or be converted into noradrenaline (Hornykiewicz, 1966; Goldberg, 1972). Systemic injection of FLA 63 with subsequent local injections of dopamine at the time of maximal inhibition of dopamine- $\beta$-hydroxylase (Svensson and Waldeck, 1969; Corrodi et al., 1970) did not affect the decrease of blood pressure caused by dopamine or $\alpha$-methylnoradrenaline. This suggests a direct effect of dopamine on a catecholaminergic receptor. The prior administration of haloperidol, which blocks dopamine receptors preferentially to noradrenaline receptors (Van Rossum, 1966; Andèn et al., 1970; Day and Roach, 1976), failed to separate the effect of dopamine from that $\alpha$-methylnoradrenaline. Also, the $\alpha$-lytic drugs phentolamine and yohimbine both blocked the effect of dopamine as well as that of $\alpha$-methylnoradrenaline. These findings may be interpreted as indicating that the catecholamines act on an $\alpha$-receptor site. This could also fit with the finding that dopamine was less effective than were noradrenaline and adrenaline. The $\alpha$-mimetic substances investigated, however, did not decrease blood pressure dose-dependently after their microinjection into the $\mathrm{A}_{2}$-region. Neither the $\alpha$-mimetics with a relatively preferential postsynaptic effect (methoxamine and phenylephrine) or that with a relatively presynaptic effect (oxymetazoline) (Starke et al., 1975; Berthelsen and Pettinger, 1977) had a marked effect. From these data it is not possible to define the catecholaminergic structures in the $A_{2}$-region responsible for the decrease of blood pressure. Adrenaline was the most potent of the catecholamines and this may indicate an adrenoceptor type. Similarly adrenaline was reported to be more effective than noradrenaline in decreasing the blood pressure of anesthetized rats following an injection into the anterior hypothalamus (Struyker Boudier and Bekers, 1975). However this effect may also reflect the more lipophylic character of adrenaline.

An initial pressor response was caused by higher doses of noradrenaline and $\alpha$-methylnoradrenaline and by the different $\alpha$-mimetics following their application into the $\mathrm{A}_{2}$-region. After higher doses of dopamine the blood pressure decrease was delayed as was also seen with $\alpha$-methylnoradrenaline. A higher dose of noradrenaline induced a delayed and less pronounced decrease of blood pressure. In previous studies we found that the pressor response to the high doses of $\alpha$-methylnoradrenaline was enhanced following the local application of phentolamine (De Jong and Nijkamp, 1976). The pressor effect, however, appears not to be explainable by $\beta$-mimetic effects since isoprenaline had no pressor activity. Although the increase of blood pressure may be caused in part by leakage to the circulation, no correlation - either positive or negative - with the peripheral vasoconstrictor activity could be found; e.g. methoxamine caused a pronounced pressor response, while peripherally it is not a very active vasoconstrictor drug. It should be noted that a dosedependent increase in blood pressure can be evoked from the same area of the NTS by local injections of physostigmine (Zandberg and De Jong, 1977b). There are thus structures in the NTS which can cause an increase in blood pressure.

The results with clonidine show that a variable decrease in blood pressure could be observed only with relatively high doses applied to the $\mathrm{A}_{2}$-region, while still higher doses only increased blood pressure. Philippu et al. (1973) showed that, in the cat, superfusion of the NTS with clonidine had no effect on blood pressure while it diminished the pressor response evoked by stimulation of the posterior hypothalamus. Lipski et al. (1976) reported that, in rats, the decrease in blood pressure caused by the intravenous injection of clonidine was significantly diminished after ablation of the NTS. Laubie 
and Schmitt (1977) and Antonaccio and Halley (1977) found that bilateral destruction of the NTS did not significantly change the blood pressure response of clonidine in cats and dogs. These authors have shown that in contrast to the hypotensive effect the NTS was the main site of the vagally mediated bradycardia of clonidine in these species. In rats, however, we only found limited changes in heart rate after the local injection of clonidine. Thus, with respect to the effect on heart rate, there may be a difference between cats and dogs on one hand and rats on the other hand in the response to clonidine. It is possible that in rats the bradycardia caused by agents like $\alpha$-methylnoradrenaline and clonidine is mediated via a decrease in sympathetic tone more than via an increase in vagal tone. De Jong and Nijkamp (1976) found that the bradycardia induced by $\alpha$-methylnoradrenaline applied into the $\mathrm{A}_{2}$ region of the NTS was only reduced by atropine and bilateral vagotomy and the blood pressure lowering effect was enhanced. It can be concluded that in the rat the NTS is not sensitive to clonidine and appears to be of limited importance for the bradycardia caused by clonidine. It is however possible that clonidine does not act primarily on the catecholaminergic structures in the NTS but on other catecholaminergic cell groups in the ponsmedulla which project to the NTS. Szreniawski et al. (1977) found that, in rats, bilateral lesions of the locus coeruleus inhibited the blood pressure lowering effect of clonidine. Our results also suggest that the locus coeruleus may be one of the sites where clonidine is capable of decreasing blood pressure. The catecholamines, however, increased blood pressure at this site while isoprenaline decreased it. Andèn et al. (1970) reported that clonidine reduced noradrenaline turnover in the brain. A reduction of spontaneous firing of the noradrenaline containing neurons of the locus coeruleus has been observed following i.v. injection of clonidine in cats (Svensson et al., 1975) or local administration in the locus coeruleus using micro-iontophore- sis (Cedarbaum and Aghajanian, 1975). These results may be explained by a selective activation of presynaptic $\alpha$-receptors by clonidine (Starke et al., 1975).

\section{Acknowledgements}

The authors are indebted to $\mathrm{H}$. de Lang, C. Creutzburg and A.J.M. Seegers for their excellent assistance.

\section{References}

Andén, N.-E., H. Corrodi, K. Fuxe, B. Hökfelt, C. Rydin and T. Svensson, 1970, Evidence for a central noradrenaline receptor stimulation by clonidine, Life Sci. 9, 513.

Antonaccio, M.J. and J. Halley, 1977, Clonidine hypotension: lack of effect of bilateral lesions of the nucleus tractus solitary tract in anaesthetized cats, Neuropharmacol. 16, 431.

Berthelsen, S. and W.A. Pettinger, 1977, A functional basis for classification of $\alpha$-adrenergic receptors, Life Sci. 21, 595.

Cedarbaum, J.M. and G.K. Aghajanian, 1976, Noradrenergic neurons of the locus coeruleus: inhibition by epinephrine and activation by the $\alpha$-antagonist piperoxane, Brain Res. 112, 413 .

Chalmers, J.P., 1975, Brain amines and models of experimental hypertension, Circulation Res. 36, 469.

Corrodi, H., K. Fuxe, B. Hamberger and $\AA$. Ljungdahl, 1970, Studies on central and peripheral noradrenaline neurons using a new dopamine- $\beta$ hydroxylase inhibitor, European J. Pharmacol. 12,145 .

Dahlström, A. and K. Fuxe, 1964, Evidence for the existence of monoamine containing neurones in the central nervous system. I. Demonstration of monoamines in the cell bodies of brain stem neurons, Acta Physiol. Scand. 62, Suppl. 232, 1.

Day, M.D. and A.G. Roach, 1976, Cardiovascular effects of dopamine after central administration into conscious cats, Brit. J. PHarmacol. 58, 505.

De Jong, W., 1974, Noradrenaline, central inhibitory control of blood pressure and heart rate, European J. Pharmacol. 29, 179.

De Jong, W., P. Zandberg and B. Bohus, 1975, Central inhibitory noradrenergic cardiovascular control, in: Hormones, Homeostasis and the Brain, Progr. Brain Res. 42, eds. W.H. Gispen, Tj.B. van Wimersma Greidanus, B. Bohus and D. de Wied (Elsevier, Amsterdam) p. 285.

De Jong, W. and F.P. Nijkamp, 1976, Centrally induced hypotension by $\alpha$-methylnoradrenaline in 
normotensive and renal hypertensive rats, Brit. J. Pharmacol. 58, 593.

Fuxe, K., 1965, Evidence for the existence of monoamine neurons in the central nervous system. IV. Distribution of monoamine nerve terminals in the central nervous system, Acta Physiol. Scand. 64, Suppl. 247, 37.

Goldberg, L.I., 1972, Cardiovascular and renal actions of dopamine, Pharmacol. Rev. 24, 1.

Haeusler, G., 1975, Cardiovascular regulation of central adrenergic mechanisms and its alteration by hypotensive drugs, Circulation Res., Suppl. I to Vols. 36 and 37,223 .

Haeusler, G., 1977, Neuronal mechanisms influencing transmission in the baroreceptor reflex arc., in: Hypertension and Brain Mechanisms, Progr. Brain Res. 47, eds. W. De Jong, A.P. Provoost and A.P. Shapiro (Elsevier, Amsterdam) p. 95.

Henning, M., 1973, Receptor regulation studies: significance of central catecholamine receptors in cardiovascular control, in: Frontiers in Catecholamine Research, ed. E. Usdin and S.H. Snyder (Pergamon Press, Oxford-London) p. 595.

Henning, M., 1975, Central sympathetic transmitters and hypertension, Clin. Sci. Mol. Med. 48, 195s.

Hornykiewicz, O., 1966, Dopamine and brain function, Pharmacol. Rev. 18, 925 .

Kobinger, W. and L. Pichler, 1975, The central modulatory effects of clonidine on the cardiodepressor reflex after suppression of synthesis and storage of noradrenaline, European J. Pharmacol. 30,56 .

König, J.F.R. and R.A. Klippel, 1963, The rat brain: a stereotaxic atlas of the forebrain and lower parts of the brainstem (The Williams and Wilkins Company, Baltimore).

Laubie, M., B. Delbarre, D. Bogaievsky, Y. Bogaievsky, D. Tsoucaris-Kupfer, D. Senon, H. Schmitt and $H$. Schmitt, 1977, Pharmacological evidence for a central $\alpha$-sympathomimetic mechanism controlling blood pressure and heart rate, Circulation Res. 38, Suppl. Il p. 35.

Laubie, M. and H. Schmitt, 1977, Sites of action of clonidine: Centrally mediated increase in vagal tone, centrally mediated hypotensive and sympatho-inhibitory effects, in: Hypertension and Brain Mechanisms, Progr. Brain Res. 47, eds. W. De Jong, A.P. Provoost and A.P. Shapiro (Elsevier, Amsterdam) p. 337 .

Lipski, J., J. Przybylski and E. Solnicka, 1976, Reduced hypotensive effect of clonidine after lesions of area of nucleus of tractus solitarii in rats, European J. Pharmacol. 38, 19.

Nijkamp, F.P. and W. De Jong, 1975, $\alpha$-Methylnoradrenaline induced hypotension and bradycardia after administration into the area of the nucleus tractus solitarii, European J. Pharmacol. 32,361 .
Palkovits, M. and D.M. Jacobowitz, 1974, Topographic atlas of catecholamine and acetylcholinesterase containing neurons in the rat brain. II. Hindbrain (mesencephalon, rhombencephalon), J. Comp. Neurol. 157, 29.

Palkovits, M. and L. Záborszky, 1977, Neuroanatomy of central cardiovascular control. Nucleus tractus solitarii: afferent and efferent neuronal connections in relation to the baroreceptor reflex arc, in: Hypertension and Brain Mechanisms, Progr. Brain Res. 47, eds. W. De Jong, A.P. Provoost and A.P. Shapiro (Elsevier, Amsterdam) p.9.

Philippu, A., W. Roensberg and H. Przuntek, 1973, Effects of adrenergic drugs on pressor responses to hypothalamic stimulation, N.S. Arch. Pharmacol. 278,373 .

Schmitt, H., H. Schmitt, S. Fénard and M. Laubie, 1973, Evidence for a sympathomimetic component inhibiting the sympathetic centres: nature of the receptor, in: Symposium on Pharmacological Agents and Biogenic Amines in the Central Nervous System, eds. J. Knoll and K. Magyar (Akademiai Kiadó, Budapest) p. 177.

Sinha, J.N., J.M. Atkinson and H. Schmitt, 1973, Effects of clonidine and l-dopa on spontaneous and evoked splanchnic nerve discharges, European J. Pharmacol. 24, 113.

Starke, K., T. Endo and H.D. Taube, 1975, Relative pre- and postsynaptic potencies of $\alpha$-adrenoceptor agonists in the rabbit pulmonary artery, N.S. Arch. Pharmacol. 291, 55.

Struyker Boudier, H.A.J. and A. Bekers, 1975, Adrenaline-induced cardiovascular changes after intrahypothalamic administration to rats, European J. Pharmacol. 31, 153.

Svensson, T., B.S. Bunney and G.K. Aghajanian, 1975, Inhibition of both noradrenergic and serotonergic neurons in brain by the $\alpha$-adrenergic agonist clonidine, Brain Res. 92, 291.

Svensson, T. and B. Waldeck, 1969, On the significance of central noradrenaline for motor activity: experiments with a new dopamine $\beta$-hydroxylase inhibitor, European J. Pharmacol. 7, 278.

Szreniawski, Z., W. Kostowski and E. Widy-Tyszkiewicz, 1976, Effects of lesions of monoaminergic systems in the brain on the hypotensive effects of clonidine in rats, Pol. J. Pharmacol. Pharm. 28, 605.

Van der Gugten, J., M. Palkovits, H.L.J.M. Wijnen and D.H.G. Versteeg, 1976, Regional distribution of adrenaline in rat brain, Brain Res. 107, 171.

Van Rossum, J.M., 1966, The significance of dopamine-receptor blockade for the mechanism of action of neuroleptic drugs, Arch. Intern. Pharmacodyn. 160, 492 .

Van Zwieten, P.A., 1975, Antihypertensive drugs with a central action, Progr. Pharmacol. 1, 1. 
Versteeg, D.H.G., J. van der Gugten, W. De Jong and M. Palkovits, 1976, Regional concentrations of noradrenaline and dopamine in rat brain, Brain Res. 113, 563.

Zandberg, P. and W. De Jong, 1977a, $\alpha$-Methylnoradrenaline-induced hypotension in the nucleus tractus solitarii of the rat: a localization study, Neuropharmacol. 16, 219.

Zandberg, P. and W. De Jong, $1977 \mathrm{~b}$, Localization of catecholaminergic receptor sites in the nucleus tractus solitarii involved in the regulation of arterial blood, pressure, in: Hypertension and Brain Mechanisms, Progr. Brain Res. 47, ed. W. De Jong, A.P. Provoost and A.P. Shapiro (Elsevier, Amsterdam) p. 117.

Zandberg, P., M. Palkovits and W. De Jong, 1978, Effect of various lesions in the nucleus tractus solitarii of the rat on blood pressure, heart rate and cardiavascular reflex responses Clin. Exptl. Hypertension (in press). 